\title{
The eclipsing bursting X-ray binary EXO 0748-676 revisited by XMM-Newton
}

\author{
J. M. Bonnet-Bidaud ${ }^{1}$, F. Haberl ${ }^{2}$, P. Ferrando ${ }^{1}$, \\ P. J. Bennie ${ }^{3}$, and E. Kendziorra ${ }^{4}$
}

1 Service d'Astrophysique, DSM/DAPNIA/SAp, CE Saclay, 91191 Gif-sur-Yvette Cedex, France

2 Max-Planck-Institut für Extraterrestrische Physik, Giessenbachstrasse, 85748 Garching, Germany

3 Department of Physics \& Astronomy, University of Leicester, University Road, Leicester LE1 7RH, UK

4 Institut für Astronomie und Astrophysik - Astronomie, University of Tübingen, Waldhäuser Strasse 64, 72076 Tübingen, Germany

Received 13 October 2000 / Accepted 8 November 2000

\begin{abstract}
The bright eclipsing and bursting low-mass X-ray binary EXO 0748-676 has been observed at several occasions by $X M M-N e w t o n$ during the initial calibration and performance verification (CAL/PV) phase. We present here the results obtained from observations with the EPIC cameras. Apart from several type-I X-ray bursts, the source shows a high degree of variability with the presence of soft flares. The wide energy coverage and high sensitivity of $X M M$-Newton allows for the first time a detailed description of the spectral variability. The source is found to be the superposition of a central $\left(\sim 210^{8} \mathrm{~cm}\right)$ Comptonized emission, most probably a corona surrounding the inner edge of an accretion disk, associated with a more extended $\left(\sim 310^{10} \mathrm{~cm}\right)$ thermal halo at a typical temperature of $\sim 0.6 \mathrm{keV}$ with an indication of non-solar abundances. Most of the variations of the source can be accounted for by a variable absorption affecting only the central comptonized component and reaching up to $N_{\mathrm{H}} \sim 1.310^{23} \mathrm{~cm}^{-2}$. The characteristics of the surrounding halo are found compatible with an irradiated atmosphere of an accretion disc which intercepts the central emission due to the system high inclination.
\end{abstract}

Key words. stars: individual (EXO 0748-676/UY Vol) - binaries: eclipsing - X-rays: stars - accretion: accretion disc

\section{Introduction}

The X-ray binary EXO $0748-676$ is unique in showing all types of variability commonly seen in different low-mass X-ray binaries. The source was discovered in outburst by the EXOSAT satellite. The observations revealed the existence of sharp eclipses, intensity dips as well as repetitive type I bursts which were used to infer the presence of a neutron star as the compact object and to estimate the distance to $\sim 10 \mathrm{kpc}$ (Parmar et al. 1986 hereafter PA86, Gottwald et al. 1986). More recent observations with the Rossi X-ray Timing Explorer (RXTE) have also shown that the source exhibits variable $(\sim \mathrm{Hz}$ and $\sim \mathrm{kHz})$ quasi-periodic oscillations (Homan et al. 1999; Homan \& van der Klis 2000). The length of the orbital period $(3.82 \mathrm{hr})$, combined with the duration of the eclipses, allows the geometry of the system to be constrained with a high $\sim 75^{\circ}$ inclination and a $\sim 0.5 M_{\odot}$ companion. The presence of residual flux during eclipses and of dips in the orbital light curve makes EXO 0748-676 typical of

Send offprint requests to: J. M. Bonnet-Bidaud, e-mail: bobi@discovery.saclay.cea.fr the ADC (accretion disc corona) sources in which the central compact source is surrounded by an asymmetric disc with the presence of a bulge in the outer edge and an extended central hot region (White \& Holt 1982). This complex geometry has prevented so far the different components to be isolated unambiguously and contradictory interpretations of the spectral variability have been put forward. From EXOSAT data, Parmar et al. (1986) concluded to the presence of a cut-off power-law emission whose part was strongly absorbed. However from ASCA observations, Church et al. (1998) described the spectrum as the superposition of a point source $(\sim 2 \mathrm{keV})$ blackbody from the neutron star and a partially covered Comptonized emission, originating from the ADC.

The very rich variability of EXO 0748-676 makes it an ideal target for XMM-Newton. The high throughput and large energy coverage of the EPIC cameras provide for the first time a detailed picture of the system. We present here the results obtained with the EPIC MOS and PN cameras. More results on the RGS Grating observations of the source can be found in Cottam et al. (2001). 
Table 1. Log of EPIC observations

\begin{tabular}{llccr}
\hline Instrument & Mode $^{1} /$ Filter & SR $^{2}$ & $\begin{array}{c}\text { Start time } \\
(2000, \mathrm{UT})\end{array}$ & $\begin{array}{r}T_{\text {exp }} \\
(\mathrm{ks})\end{array}$ \\
\hline MOS1+2 & FF/Thin & 59 & Apr. 04, 17:18 & 20.0 \\
MOS1+2 & FF/Medium & 67 & Apr. 21, 04:11 & 16.9 \\
PN & FF/Medium & 67 & Apr. 21, 03:59 & 18.1 \\
PN & LW/Medium & 67 & Apr. 21, 09:50 & 3.2 \\
PN & SW/Medium & 67 & Apr. 21, 15:22 & 18.1 \\
\hline
\end{tabular}

(1) FF Full Frame, LW Large and SW small window mode.

(2) Satellite revolution.

\section{Observations and data reduction}

EXO 0748-676 was observed by XMM-Newton at four occasions during the $\mathrm{CAL} / \mathrm{PV}$ phase, in revolutions 50,55 , 59 and 67 of the satellite. Details on the XMM-Newton mission and on the EPIC MOS and PN cameras can be found in Jansen et al. (2001), Turner et al. (2001) and Strüder et al. (2001).

Scientific data from EPIC MOS were secured in revolutions 59 and 67 (see Table 1). The data, obtained with a $2.6 \mathrm{~s}$ time resolution, were processed with the standard analysis of the XMM Science Analysis System. This involves the removal of bad or hot pixels, and of the electronic noise. For the MOS, X-ray events with pattern 0 to 12 (similar to grades 0-4 in ASCA) were selected. The gain was adjusted with reference to the closest observation in the Closed Calibration position. The source events were selected by applying a mask of radius $1.5^{\prime}$ centered on the source which corresponds to $\sim 98 \%$ of the energy of the typical point spread function of the telescope (Gondoin et al. 2000). The source is bright and some effects of the pile-up of events might be expected in the FF mode. The average counting rate in each MOS camera, except for the bursts, was found to be between (7-10) event/reading frame which is at the threshold for significant pile-up (see Fig. 6 in Ballet 1999). The pile-up rate is expected to be less than $\sim 0.8 \%$ so that a moderate effect should affect the most central part of the image.

EPIC PN data are currently available from revolution 67 of XMM-Newton in three different modes (Table 1). For light curves we selected the valid event-patterns (single, double, triple and quadruple; pattern 0-12) while for the spectral analysis only single-events (pattern 0) were used for which an advanced spectral response matrix exists. To avoid pile-up effects only the data from the fastest readout mode (the SW) was used for the spectral analysis.

\section{The varying light curve of EXO 0748-676}

\subsection{The EPIC MOS observations}

Light curves were produced by selecting the source events in two different soft $S(0.5-2 \mathrm{keV})$ and hard $H(2-10 \mathrm{keV})$ energy ranges and adding the counts from the two MOS cameras. The background rate is found negligible with mean values of $\sim 0.04$ and $\sim 0.08$ counts $\mathrm{s}^{-1}$, respectively in the soft and hard bands, illustrating the very clean images obtained by XMM-Newton. Figures 1 and 2 show the extreme variability of the source for the two observations. In each observation, an eclipse is clearly seen in the hard band as well as several type I bursts with counting rates reaching up to 25 counts $\mathrm{s}^{-1}$.

The characteristics of the two eclipses are similar to those reported from EXOSAT (PA86) and RXTE (Hertz et al. 1997). The present lack of exact absolute timing prevents a precise comparison with the source ephemeris. However, the use of the provisional start times listed in Table 1 gives observed mid eclipses times which fall within $17 \mathrm{~s}$ (April 4) and $38 \mathrm{~s}$ (April 21) of the predicted times using the linear ephemeris of Hertz et al. (1997). More tight constraints on the period changes will be provided when precise absolute timings will be available. Due to the very low background rates, the residual counts in eclipse can be measured for the first time very accurately. In the hard band, the residual counts are $(0.50 \pm 0.20)$ counts s $^{-1}$ and $(0.37 \pm 0.18)$ counts $\mathrm{s}^{-1}$ on April 4 and 21 respectively, which corresponds to $(10 \pm 4) \%$ and $(7 \pm 3) \%$ of the corresponding mean flux outside eclipse. Significantly, this ratio, in the time interval corresponding to the hard eclipse, is $(87 \pm 25) \%$ and $(32 \pm 10) \%$ in the soft band. The behaviour of the source is different in the two observations as seen in Figs. 1 and 2.

On 4 April 2000, the variability is mainly confined to the hard band with the presence of two typical dips, around phase $\sim 0.7$ of the $3.82 \mathrm{hr}$ cycle (where phase 0 is defined as the mid-eclipse time). There is no evidence in the soft band of the eclipse nor of the dips. The hardness ratio, computed as $(H-S) /(H+S)$, is also shown at the top of Figs. 1 and 2. A significant softening is seen during eclipse, bursts and dips. On 21 April 2000, the source appears in a quite different and more active state. An intense flaring activity is seen in the soft band with two major increases of flux arising around phase 0.2 and 0.6. The hardness ratio is marked by a significant softening during these flares.

A similar activity around the same phases has been noted from previous ASCA observations though above $1 \mathrm{keV}$ and with somewhat different energy bands (Church et al. 1998). The lack of information at lower energy has made the interpretation uneasy in this case. The very wide spectral coverage of EPIC allows here a more straightforward separation between the lowest energies (below $2 \mathrm{keV}$ ) where the absorption effects are dominant and the higher ones (above $2 \mathrm{keV}$ ) where they are negligible (see below). We note also that our definition of the hardness ratio is quite different to that adopted in the $1-10 \mathrm{keV}$ range for the EXOSAT observations (PA86). 

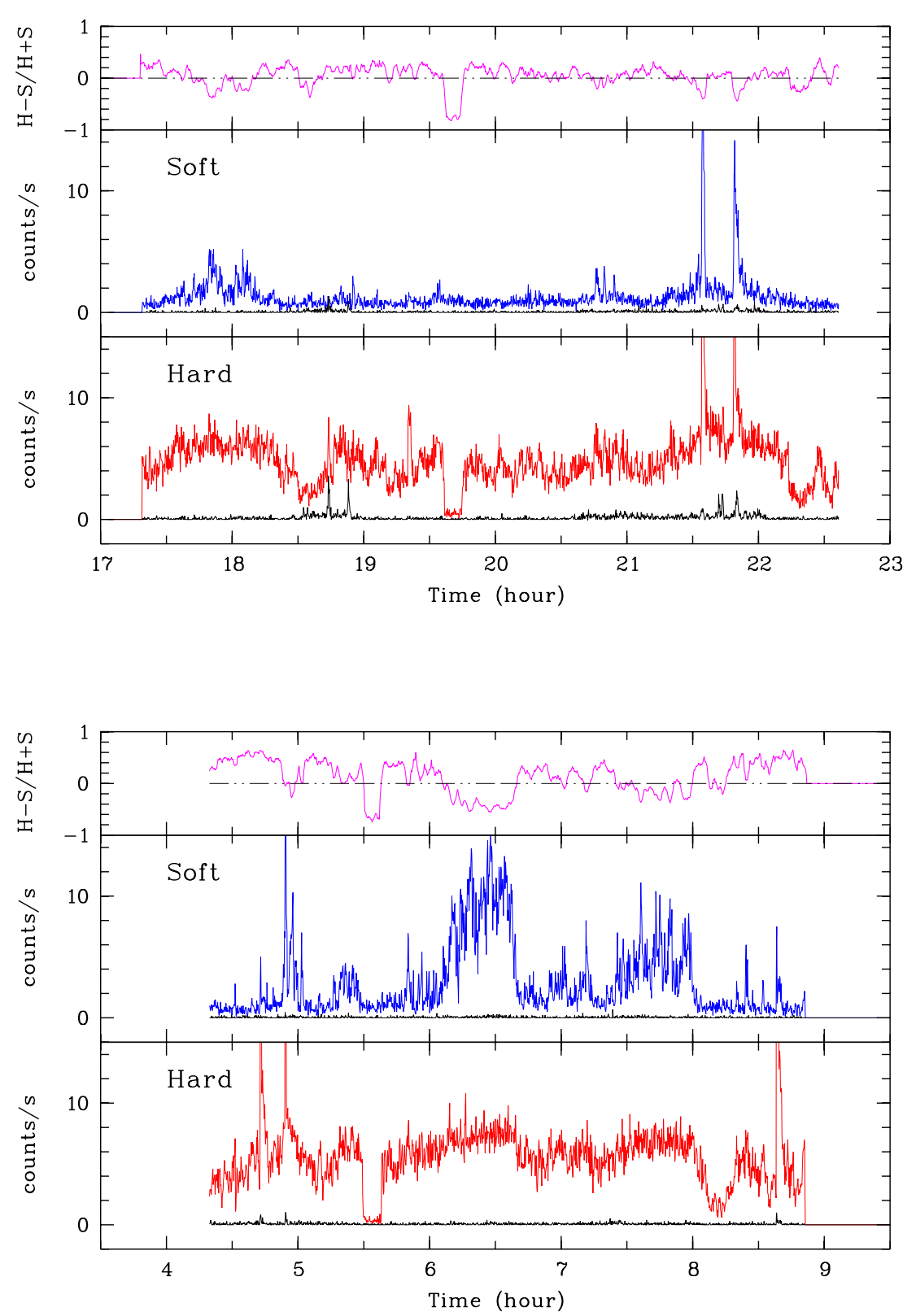

Fig. 1. The MOS hard $H(2-10 \mathrm{keV})$ (bottom) and soft $S(0.5-2 \mathrm{keV})$ (middle) light curves of EXO 0748-676 as observed by XMM-Newton on 2000 April 4. Time in hours from 2000 April 4, 0:0 UT. The curves are source counts obtained from the two MOS cameras and binned to $10 \mathrm{~s}$. In each case, the underlying curve is the background rate accumulated in a region of the image free of sources. The hardness ratio computed as $(H-S) /(H+S)$ is shown on the top, where the curve have been smoothed by a 5 -points average. Note the near total eclipse at $\sim 19: 40$ UT and the two dips at $\sim 18: 30$ UT and $\sim 22: 20$ UT. Burst peak counts are cut in the figure
Fig. 2. The MOS light curves for 2000 April 21, with the same scale as Fig. 1. Time in hours from 2000 April 21, 0:0 UT. Note the two significant soft flares around 06:30 UT and 07:40 UT

\subsection{The EPIC PN observations}

The EPIC PN observations of EXO 0748-676 in FF and SW modes on April 21 (see Table 1) were performed accidentally at almost identical binary phases. The light curves are qualitatively identical showing marked features like flaring activity and dips at similar phases. Since during the FF observation also the MOS cameras observed EXO 0748-676 (Fig. 2) we show only the EPIC PN light curves of the SW observation in Fig. 3.

\section{Spectral variability}

Source spectra were accumulated from the EPIC PN data, using a circular extraction region of radius $40^{\prime \prime}$ around the peak surface brightness. Background spectra were created in adjacent regions of the same size. In view of the large variability of the source four representative spectra were chosen by selecting events in time intervals corresponding to the first and second soft flaring episodes (F1 and F2), the between-flare or quiescent interval (Q) and the dip part (D) of the EPIC PN SW observation on April 21 (see Fig. 3). The count rate during this observation was well below the pile-up limit of the SW mode of $\sim 100$ counts s $^{-1}$. Spectra were binned to obtain at least 20 counts per bin. The most recent response matrices (version Sept. 2000) were obtained from the hardware groups and the spectra analyzed using the XSPEC package.

Descriptions with a power-law and either a blackbody or a multi-temperature blackbody accretion disc model, as suggested from previous observations, were found 


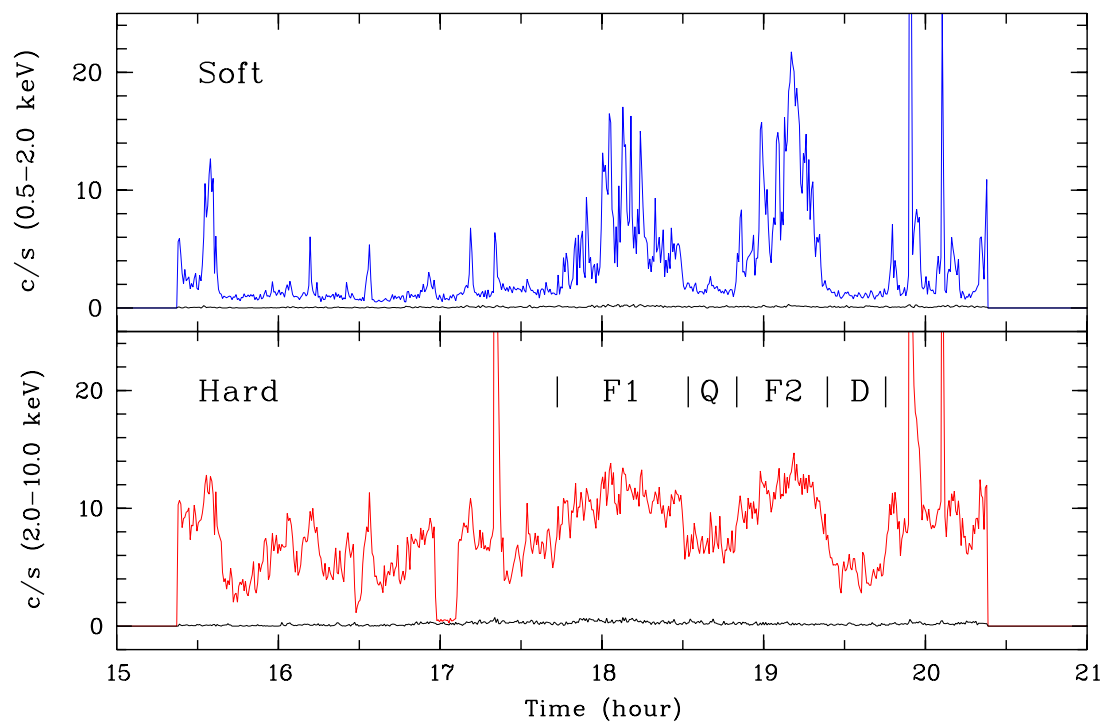

Fig. 3. EPIC PN light curves of EXO 0748-676 from the small window mode observation on 2000 April 21 in the $0.5-2.0 \mathrm{keV}$ (top) and $2.0-10.0 \mathrm{keV}$ energy bands. Data binning is $30 \mathrm{~s}$ and time in hours from 2000 April 21, 0:0 UT. The background contribution is between 0.05 and 0.2 counts $\mathrm{s}^{-1}$ in the soft band and $0.05-0.5$ counts $\mathrm{s}^{-1}$ in the hard band. Note the similarity of the light curves during different binary orbits (see Fig. 2), in particular the stable phasing of the flaring events

inadequate (see Table 2 where reduced $\chi^{2}$ values are given for various spectral models). In particular none of the models represents the spectrum around $0.7 \mathrm{keV}$ where a steep decline due to an emission line feature can be seen. This line can be attributed to the O-VIII ion. To model the soft part of the spectra below $2 \mathrm{keV}$ where more lines are visible we therefore tried thermal emission models, keeping in mind that the models available in XSPEC were developed for a thin plasma in collisional equilibrium, while the plasma which likely exists in EXO 0748-676 is photoionized by hard X-ray emission from a central source (see below).

Best fits in the range $0.15-10 \mathrm{keV}$ were obtained with a combination of a power-law (PL) and a Raymond-Smith thermal component (RS), each attenuated by individual photo-electric absorption (Morrison \& McCammon 1983). Elemental abundances were treated relative to solar (Anders \& Grevesse 1989). It was checked that the fits with the Mewe et al. (1985) instead of the RS thermal model gives fully consistent results including for the abundances.

The four representative spectra were jointly fitted by the same model. In the power-law component, intensity and index were each kept as single free parameter tied together for all four spectra. Only the column density attenuating this component was allowed to vary individually. Similarly in the thermal component, the temperature and the elemental abundances were tied, but intensity and absorption allowed to vary separately. The abundances of Si and higher $Z$ elements were fixed to zero as is suggested by the RGS spectrum where no emission lines of these elements are found (Cottam et al. 2001). The other abundances were allowed to vary freely. The best fit RS model results in non-solar abundances of $\mathrm{N}(10 \pm 2)$, $\mathrm{O}(0.6 \pm 0.1), \mathrm{Ne}(0.02 \pm 0.02)$ and $\mathrm{Mg}(0.31 \pm 0.23)$. The $\mathrm{Mg}$ line was only seen in the spectrum between flares. Table 3
Table 2. Model fits to the flare spectrum (F1)

\begin{tabular}{lc}
\hline \multicolumn{1}{c}{ Model $^{1}$} & $\chi_{\mathrm{r}}^{2} /$ dof \\
\hline powerlaw*wabs & $6.66 / 1071$ \\
(powerlaw+bbody)*wabs & $2.10 / 1069$ \\
powerlaw*wabs+bbody*wabs $^{*}$ & $1.57 / 1068$ \\
powerlaw*wabs+diskbb*wabs & $1.62 / 1068$ \\
\hline
\end{tabular}

(1) Model components as defined in XSPEC.

summarizes the best fit continuum parameters for the different intensity levels of the source. All quoted errors were determined at $\Delta \chi^{2}$ of +2.7 around the $\chi^{2}$ minimum. The spectra are shown in Fig. 4 with superimposed best fit spectral models. Residual features around emission lines seen below $1 \mathrm{keV}$ are caused by calibration problems of the charge transfer losses in SW mode. In the current version of charge transfer loss correction in SW the event energies are over-corrected by about $10-20 \mathrm{eV}$ at energies below $1 \mathrm{keV}$, i.e. the data points in the spectra are shifted to the right. This causes the typical residual pattern seen in the bottom panel of Fig. 4 where data and model emission lines are shifted slightly against each other in energy. This should affect the derived abundances only slightly and will not affect our conclusions. Other residuals near $2.2 \mathrm{keV}$ are caused by problems in the effective mirror areas around the Au edge.

The modulation of the source is mainly due to a strong PL absorption varying from $13.010^{22} \mathrm{~cm}^{-2}$ in the dip to $\sim 610^{22} \mathrm{~cm}^{-2}$ in quiescence and down to (1.5-2.2) $10^{22} \mathrm{~cm}^{-2}$ during flares. In the same time, the soft $\mathrm{RS}$ component is affected by a much lower (0.04-0.1) $10^{22} \mathrm{~cm}^{-2}$ absorption increasing only to $0.210^{22} \mathrm{~cm}^{-2}$ during the dip. However, the flares are marked also by a significant increase in the RS intensity. 
Table 3. Best-fitting spectral parameters

\begin{tabular}{lccccrrr}
\hline & \multicolumn{3}{c}{ Power-law } & \multicolumn{3}{c}{ Thermal component } \\
& $A_{\mathrm{PL}}\left(^{*}\right)$ & $\alpha\left(^{*}\right)$ & $N_{\mathrm{H}}$ & $A_{\mathrm{RS}}$ & $k T(\mathrm{keV})\left(^{*}\right)$ & \multicolumn{1}{c}{$N_{\mathrm{H}}$} & $\chi_{\mathrm{r}}^{2} / \mathrm{dof}$ \\
\hline Flare 1 & $0.0230(12)$ & $1.35(3)$ & $2.25(11)$ & $0.021(3)$ & $0.64(5)$ & $0.041(6)$ & $1.27 / 2467$ \\
Quiescent & & & $6.43(28)$ & $0.009(2)$ & & $0.145(26)$ & \\
Flare 2 & & & $1.48(10)$ & $0.033(4)$ & & $0.044(6)$ & \\
Dip & & & $13.1(6)$ & $0.011(2)$ & & $0.22(3)$ & \\
\hline
\end{tabular}

$\left(^{*}\right)$ single parameter in the joint fit to all four spectra.

$A_{\mathrm{PL}}$ in photons $\mathrm{cm}^{-2} \mathrm{~s}^{-1} \mathrm{keV}^{-1}$ at $1 \mathrm{keV}, \alpha$ photon index, $N_{\mathrm{H}}$ in units of $10^{22} \mathrm{~cm}^{-2}$.

$A_{\mathrm{RS}}$ emission measure in units of $10^{-14} /\left(4 \pi d^{2}\right) \int n_{\mathrm{e}} n_{\mathrm{H}} \mathrm{d} V$, where $d$ is the distance to the source $(\mathrm{cm})$, $n_{\mathrm{e}}$ and $n_{\mathrm{H}}$ are the electron and hydrogen densities $\left(\mathrm{cm}^{-3}\right)$.

EX00748-676 00670123500101 PNS003 SW

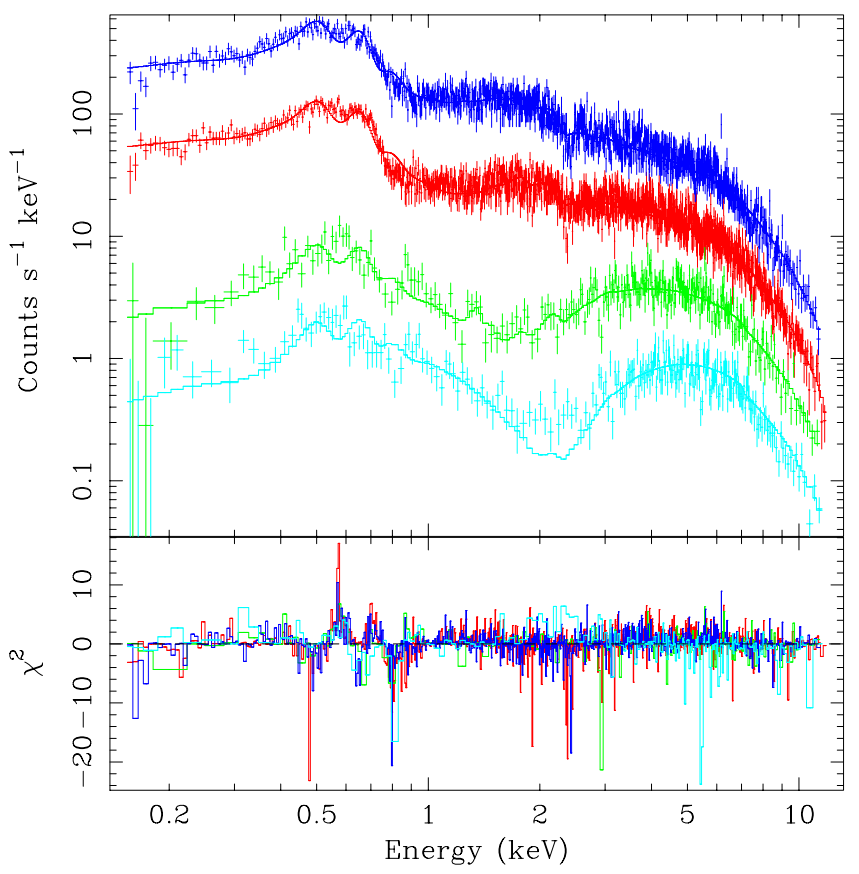

Fig. 4. The XMM-Newton spectrum of EXO 0748-676. Spectra of four different parts of the orbital light curve are shown such as flaring episodes F1 (red) and F2 (blue), interflare Q (green) and dip D (light blue). The spectra were multiplied by different factors to separate them in the plot (F1 9.0, F2 27.0, Q 3.0, D 1.0). The best fitting model (histograms) is a combination of a highly absorbed power-law and a thin plasma $(0.6 \mathrm{keV})$ thermal emission component with non-solar abundances and separate (smaller) absorption. The bottom panel shows the contribution of the residuals to the total $\chi^{2}$. The spectra are reproduced by varying mainly the amount of absorption in the power-law component. Note that the intrinsic power-law intensity is constant, i.e. the spectra at the high energy end, when not scaled, lie on top of each other

\section{Discussion}

The very good sensitivity of the EPIC cameras at energies below $2 \mathrm{keV}$ allows for the first time to build a consistent picture of the source. A straightforward interpretation of its variability can be made with an unique effect of absorption in front of a stable underlying emission with two separated components. The hard component, best described by a power-law, is suggestive of a Comptonizedtype spectrum with a high energy break high above the $12 \mathrm{keV} X M M-N e w t o n$ limit. This same component was also identified in EXOSAT and ASCA observations (PA86, Church et al. 1998). However at low energy, the XMMNewton observations show clearly that the emission is dominated by a thin hot plasma, affected by a much lower absorption than the hard component. This is at variance with previous interpretations involving a second unabsorbed Comptonized spectrum (PA86) or a blackbody emission (Church et al. 1998).

A constraint on the size of the emission area for the hard Comptonized emission is provided by its nearly complete eclipse in the orbital cycle. For a binary orbit with a $P_{\text {orb }}=3.82 \mathrm{~h}$, the transition time $(\delta T)$ in and out of eclipses yields a typical size of $R=5.4110^{11}$ $\left(M_{x}+M_{\mathrm{c}}\right)^{1 / 3} \cdot\left(\delta T / P_{\mathrm{orb}}\right) \cdot \sin i \mathrm{~cm}$, where $M_{x}$ and $M_{\mathrm{c}}$ are the masses of the compact star and of the companion in $M_{\odot}$ and $i$ the inclination of the system. Eclipse transition times are best determined by high time resolution data of 19 eclipses seen by EXOSAT (PA86) and 10 eclipses seen by RXTE (Hertz et al. 1997) which give mean values of $\sim 6 \mathrm{~s}$ (EXOSAT) and $\sim 5 \mathrm{~s}$ (RXTE). Assuming a compact star of $1.0 M_{\odot}$, a $0.45 M_{\odot}$ companion and an inclination of $i=75^{\circ}$ (PA86), yields a typical radius of the Comptonized emission of $R=(2.4 \pm 0.5) 10^{8} \mathrm{~cm}$.

On the other hand, the soft thermal component is seen un-eclipsed so that its size should be significantly greater than the companion occulting sphere which, for a Roche lobe filling star on the main sequence is of radius $R \sim$ $7.910^{9} \cdot P_{\text {orb }}(\mathrm{hr}) \mathrm{cm}$ (Frank et al. 1992). This corresponds to $\sim 310^{10} \mathrm{~cm}$ for EXO 0748-676.

The picture of the system as seen by XMM-Newton is therefore a rather compact $\left(\sim 210^{8} \mathrm{~cm}\right)$ Comptonized region as a central hard X-ray source surrounded by a more extended $\left(\sim 310^{10} \mathrm{~cm}\right)$ hot halo-type thermal emission. This is the opposite to the previous descriptions in which the central source was expected to be a $(\sim 2 \mathrm{keV})$ blackbody surrounded by a Comptonized emission from the accretion disc corona (ADC) (Church et al. 1998). 
We stress that the inner Comptonized region is compatible in size with the disturbed inner edge of the accretion disc where low frequency QPOs are expected to form. If the observed (0.58-2.44) Hz QPOs (Homan et al. 1999) are indeed produced at the Keplerian frequency in the accretion disc, their distance to the centre is given by $r=1.5010^{8} \nu^{-2 / 3} \cdot\left(M_{x} / M_{\odot}\right) \mathrm{cm}$, where $\nu$ is the QPO frequency, which corresponds to a range of $(0.8-2.2) 10^{8} \mathrm{~cm}$ in radial distance. The $\mathrm{ADC}$ is then strictly restricted to the central part of the disc. The spectral variability demonstrates that we always see this inner ADC strongly absorbed even during flares, most probably because of the high inclination and of the occultation by the external parts of the disc including the bulge during the dip.

The most extended halo region is much less absorbed. In fact we note that the lowest best fitting value of $N_{\mathrm{H}}$ is a factor two lower than the average galactic column density in this direction $N_{\mathrm{H}} \sim 1.110^{21} \mathrm{~cm}^{-2}$ (Dickey \& Lockman 1998). This is possibly due to the more complex shape of the continuum in this region and a contamination by highly ionized regions in the system which should be accounted for by a warm absorber. The absorption during the dip is a factor $\sim 2$ higher than the galactic value, revealing that the thermal extended halo is also significantly affected by absorption of the bulge. This is in favour of a flattened rather than spherical halo. The emission measure of this thermal halo in quiescent state is $\sim 0.810^{58} \mathrm{~cm}^{-3}$ for a $10 \mathrm{kpc}$ distance. For a spherical halo, with a typical dimension of $\sim 310^{10} \mathrm{~cm}$, the electron density will then be $n \sim 0.810^{13} \mathrm{~cm}^{-3}$. If a flat geometry is assumed, with a radius of $\sim 310^{10} \mathrm{~cm}$ and a typical height of $h \sim 10^{9} \mathrm{~cm}$, this density is significantly higher at $n \sim 5.310^{14} \mathrm{~cm}^{-3}$. We note that very similar values of emission measures and densities are derived from the characteristics of the absorption edges and emission lines of highly ionized elements clearly detected by the RGS at energies lower than $2.5 \mathrm{keV}$ (Cottam et al. 2001). If the central source is seen through such a flat halo, a rough estimate of the absorption is $N_{\mathrm{H}} \sim h . n \sim 5.310^{22} \mathrm{~cm}^{-2}$. This value is consistent with the measured absorption of $6.410^{22} \mathrm{~cm}^{-2}$ (Table 3 ). The estimated dimension and density of the halo suggest that it may be a hot atmosphere at the surface of an irradiated accretion disc. The high inclination of the system will impose then that the central source is always seen through this extended highly ionized region on top of the accretion disc. A better description of the halo may therefore be in terms of a fully photo-ionized model which is beyond the scope of this letter. Some caution should then be taken in interpreting the abundances derived from a pure collisional RS model.

Assuming a distance of $\sim 10 \mathrm{kpc}$, consistent with a maximum Eddington luminosity in the bursts (Gottwald et al. 1986), the $(2-10 \mathrm{keV})$ unabsorbed luminosity of the source is constant at $\sim 2.010^{36} \mathrm{erg} \mathrm{s}^{-1}$ which is comparable to the luminosities observed by ASCA and EXOSAT, though at the lowest end. In the total $X M M-N e w t o n$ range, the $(0.5-10 \mathrm{keV})$ luminosity only slightly varies from 2.64 (in quiescence) to $2.7910^{36} \mathrm{erg} \mathrm{s}^{-1}$ (flares). In this range, the thermal halo luminosity varies from 0.6 (quiescence) to $2.110^{35} \mathrm{erg} \mathrm{s}^{-1}$ (flares), contributing from 2 to $7.5 \%$ to the total luminosity. The increase of the halo luminosity is apparently not linked to any simultaneous changes in the central source luminosity. The total increase of energy inside the flares with typical duration of $\sim 2000 \mathrm{~s}$ is $\sim 310^{38} \mathrm{erg}$. This is only a small fraction of the energy $\sim 210^{39}$ erg released during the bursts. It is then possible that the halo changing luminosity may be due to a delayed re-processing of a previous burst at the surface of the accretion disc.

Acknowledgements. The results presented are based on observations obtained with XMM-Newton, an ESA science mission with instruments and contributions directly financed by ESA Member States and the USA (NASA).

EPIC was developed by the EPIC Consortium led by the Principal Investigator, Dr. M. J. L. Turner. The consortium comprises the following Institutes: University of Leicester, University of Birmingham, (UK); CEA/Saclay, IAS Orsay, CESR Toulouse, (France); IAAP Tübingen, MPE Garching, (Germany); IFC Milan, ITESRE Bologna, IAUP Palermo, (Italy). EPIC is funded by: PPARC, CEA, CNES, DLR and ASI.

\section{References}

Anders, E., \& Grevesse, N. 1989, Geochim. Cosmochim. Acta, 53, 197

Church, M., Balucinska-Church, M., Dotani, T., \& Asai, K. 1998, ApJ, 504, 516

Cottam, J., Kahn, S. M., Brinkman, A. C., den Herder, J. W., \& Erd, C. 2001, A\&A, 365, L277

Dickey, J. M., \& Lockman, F. J. 1990, ARA\&A, 28, 215

Frank, J., King, A., \& Raine, D. 1992, in Accretion Power in Astrophysics (Cambridge Univ. Press)

Gondoin, Ph., Aschenbach, B., Erd, C., et al. 2000, SPIE

Gottwald, M., Haberl, F., Parmar, A., \& White, N., 1986, ApJ, 308, 213

Hertz, P., Wood, K., \& Cominsky, L. 1997, ApJ, 486, 1000

Homan, J., Jonker, P., van der Klis, M., \& van Paradijs, I. 1999, ApJ, 516, L91

Homan, J., \& van der Klis, M., 2000, ApJ 539, 847

Jansen, F., Lumb, D., Altieri, B., et al. 2001, A\&A, 365, L1

Mewe, R., Gronenschild, E. H., \& van den Oord, G. H. 1985, A\&AS, 62, 197

Morrison, R., \& McCammon, D. 1983, ApJ, 270, 119

Parmar, A., White, N., Giommi, P., \& Gottwald, M. 1986, (PA86), ApJ, 308, 199

Strüder, L., Briel, U., Dennerl, K., et al. 2001, A\&A, 365, L18

Turner, M. J. L., Abbey, A., Arnaud, M., et al. 2001, A\&A, $365, \mathrm{~L} 27$

White, N., \& Holt, S. 1982, ApJ, 257, 318 